\title{
High-quality radio-frequency inductors on silicon using a hybrid ferrite technology
}

\author{
M. Saidani ${ }^{\mathrm{a})}$ and M. A. M. Gijs \\ Institute of Microelectronics and Microsystems, Swiss Federal Institute of Technology Lausanne, \\ CH-1015 Lausanne EPFL, Switzerland
}

(Received 13 November 2003; accepted 8 April 2004; published online 14 May 2004)

\begin{abstract}
We have realized millimeter-size rf inductors on silicon using a polyimide mold-copper electroplating coil technology. Subsequently, the coils are assembled with magnetic cover plates of commercially available bulk $\mathrm{Ni}-\mathrm{Zn}$ ferrites of high resistivity. Using the magnetic flux-amplifying ferrite plates, we obtain a $40 \%$ enhancement of the inductance and a $25 \%$ enhancement of the quality factor $(Q=10-20)$ for frequencies up to $0.2 \mathrm{GHz}$. Our results indicate the large potential of using bulk ferrites for rf applications in a hybrid inductor assembly process. (C) 2004 American Institute of Physics. [DOI: 10.1063/1.1756677]
\end{abstract}

High-performance inductors are of increasing importance for applications at $\mathrm{GHz}$ frequencies. A suitable inductor is characterized by a high inductance and quality factor and by a low resistive loss and parasitic capacitance. Use of thick and low-resistivity metals for the inductor windings obviously reduces the series resistance of the coil. Reduction of eddy current losses in the substrate can be achieved by applying appropriate dielectric layers in between the inductor coil and the normal Si substrate $\left(\rho_{\mathrm{Si}}=20-50 \Omega \mathrm{cm}\right),{ }^{1}$ by using a special high-resistivity substrate $\left(\rho_{\mathrm{Si}}>200 \Omega \mathrm{cm}\right.$, which is less compatible with transistor fabrication), ${ }^{2}$ by etching a cavity beneath the coil, ${ }^{3}$ or by positioning the inductors out of plane. ${ }^{4}$ Another approach to enhance the inductor performance is by applying a magnetic film on top of and/or below the rf coil, the function of which is to enhance and confine the magnetic flux. Such an approach has led to a quality factor $Q=7$ at $1 \mathrm{GHz}$ for $0.4 \mathrm{~mm}$ size coils, representing an enhancement of $12 \%$ over that of a conventional air-core spiral coil. ${ }^{5,6}$ More recently, values of $Q=12$, representing an enhancement of $23 \%$, have been obtained by micropatterning amorphous magnetic CoNbZr layers, sandwiching the coils. ${ }^{7}$ The latter success was based on the use of an amorphous alloy with relatively high resistivity, limiting eddy current losses, and the micropatterning of these films, giving rise to a decreased effective permeability, limiting ferromagnetic resonance losses at $\mathrm{GHz}$ frequencies. ${ }^{8}$

It is clear that, in this context, high-resistivity $\mathrm{Ni}-\mathrm{Zn}$ ferrites $\left(\rho \sim 10^{7} \Omega \mathrm{cm}\right)^{9}$ may present an interesting option for use as a magnetic material. Sputtering, ${ }^{10}$ spin coating, ${ }^{11}$ or plating ${ }^{12}$ techniques have been proposed for the realization of ferrite films, but such films often require a postdeposition annealing up to $1000^{\circ} \mathrm{C}$, which is not compatible with integrated circuit (IC) postprocessing. Pulsed laser deposition of high-quality $(\mathrm{Mn}-\mathrm{Zn}) \mathrm{Fe}$ ferrite films, having bulk magnetic properties, was achieved at $400-600{ }^{\circ} \mathrm{C}$ by a careful selection of buffer layers. ${ }^{13}$ However, there appears to be no readily available technique for fabrication of ferrite films with the desired magnetic properties at a sufficiently low

${ }^{a)}$ Electronic mail: menouer.saidani@epfl.ch temperature compatible with an IC-based process.

In this letter, we report on the microfabrication of polyimide embedded $\mathrm{Cu}$ coils on top of normal Si substrates and assembly of these coils with magnetic cover plates of commercially available bulk $\mathrm{Ni}-\mathrm{Zn}$ ferrites of high resistivity. The hybrid combination of state-of-the-art ferrites with postprocessed coils on $\mathrm{Si}$ enables one to evaluate the ultimate potential of ferrite-based inductors for rf applications. Using the magnetic flux-amplifying ferrite plates, we obtain a $40 \%$ enhancement of the inductance and a $25 \%$ enhancement of the quality factor with respect to the simple coil, for frequencies up to $0.2 \mathrm{GHz}$.

Figure 1 shows an oblique schematic view of a $\mathrm{Cu}$ coil of size $0.5 \mathrm{~mm}<d<2 \mathrm{~mm}$, fabricated on a flexible $25 \mu \mathrm{m}$ thick Kapton film, which is laminated over a $50 \Omega \mathrm{cm}$ silicon substrate. The coil consists of $15 \mu \mathrm{m}$ thick and $40 \mu \mathrm{m}$ wide $\mathrm{Cu}$ windings $(N=1-3)$ with a winding pitch of $80 \mu \mathrm{m}$ and is, except for the contact paths, fully encapsulated in polyimide PI2611 (Dupont, Germany) [see Fig. 2(a) and Ref. 14 for details on the fabrication process]. The embedded coil can be simply covered with a ferrite plate [Fig. 2(b)] and also is easily detachable from the silicon substrate [Fig. 2(c)]. We have used $1 \mathrm{~mm}$ thick and $1 \mathrm{~cm}$ wide square plates of four different $\mathrm{Ni}-\mathrm{Zn}$ ferrites listed in Table I, all with a resistivity of the order of $10^{7} \Omega \mathrm{cm}$, which are loosely positioned on

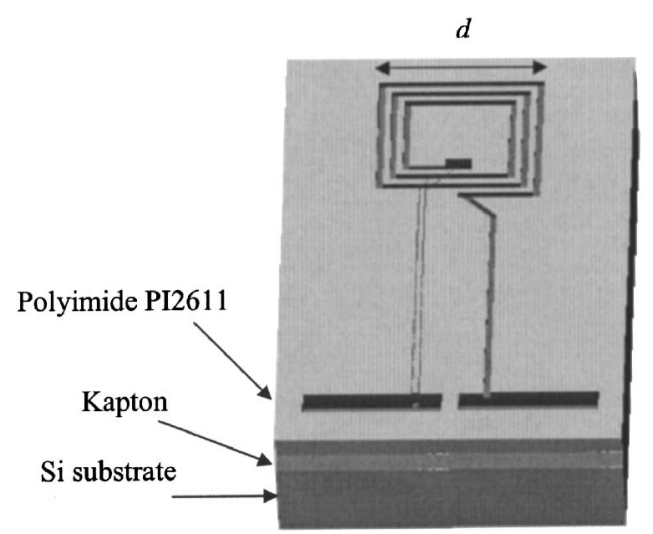

FIG. 1. Oblique schematic view of a $\mathrm{Cu}$ coil of width $d$ encapsulated in a polyimide PI2611 structure, which is realized on top of a detachable Kapton film on a Si substrate. 


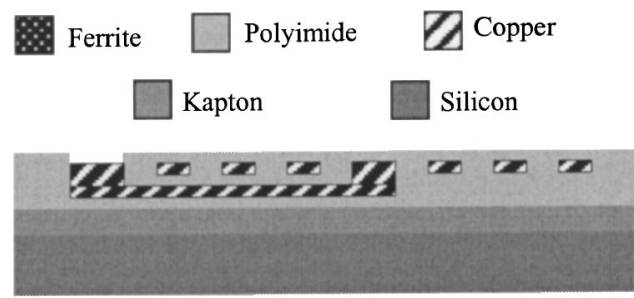

(a)

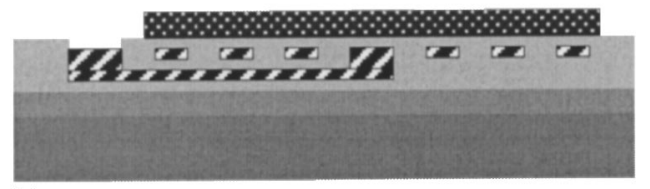

(b)

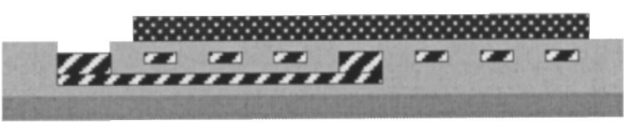

(c)

FIG. 2. Illustration of the chosen configurations: (a) Polyimide embedded coil on top of the Si substrate; (b) coil on the Si substrate with a ferrite cover plate; and (c) coil with ferrite cover plate and detached from the Si substrate.

the coils. Alternatively, such ferrite plates can be subdivided by micromachining and combined with the coils in a batchtype gluing process, similar to the process of Ref. 14. Ni-Zn ferrites are generally used for electromagnetic interference suppression applications and have an appreciable magnetic permeability up to $1 \mathrm{GHz}$, the highest frequency with still a magnetic behavior among the ferrites with cubic anisotropy. ${ }^{8}$ Their very high resistivity prevents eddy currents losses, present in metallic magnetic materials, and only magnetic resonance effects can cause dissipation at $\mathrm{GHz}$ frequencies.

We have measured the inductance, resistance, and quality factor of the inductors in the $0.001-3 \mathrm{GHz}$ range using an Agilent E4991A rf Impedance/Material Analyzer and a Cascade Microtechnique probe station. We present in this letter results for coils with an outer diameter $d=750 \mu \mathrm{m}$ and a number of turns $N=1$ and $N=3$, which are typical for our experiments. We consider the three configurations of Fig. 2. Measurements were done first on the silicon substrate with only the $\mathrm{Cu}$ coil on top [Fig. 2(a)]. Then, a ferrite cover plate was deposited on the top of the coil [Fig. 2(b)], and finally the inductor with ferrite cover was removed from the Si substrate [Fig. 2(c)]. Results for the three configurations are shown in Fig. 3.

Figure 3(a) is the frequency-dependent inductance for two coils with 1 and 3 windings, respectively. When $N=1$, we clearly observe the enhancement of the inductance due to the presence of the ferrites, irrespective from the presence of the $\mathrm{Si}$ substrate. For $N=3$, we observe a more distinct reso-

TABLE I. Magnetic permeability at $1 \mathrm{MHz}$ of the selected ferrite materials, as specified by the manufacturer.

\begin{tabular}{cc}
\hline \hline $\begin{array}{c}\text { Ferrite grade } \\
\text { (Ferroxcube) }\end{array}$ & $\begin{array}{l}\text { Initial permeability } \\
\text { @ 1 MHz }( \pm 20 \%)\end{array}$ \\
\hline $4 \mathrm{C} 65$ & 125 \\
$4 \mathrm{E} 1$ & 15 \\
$4 \mathrm{E} 2$ & 25 \\
$4 \mathrm{~F} 1$ & 80 \\
\hline \hline
\end{tabular}
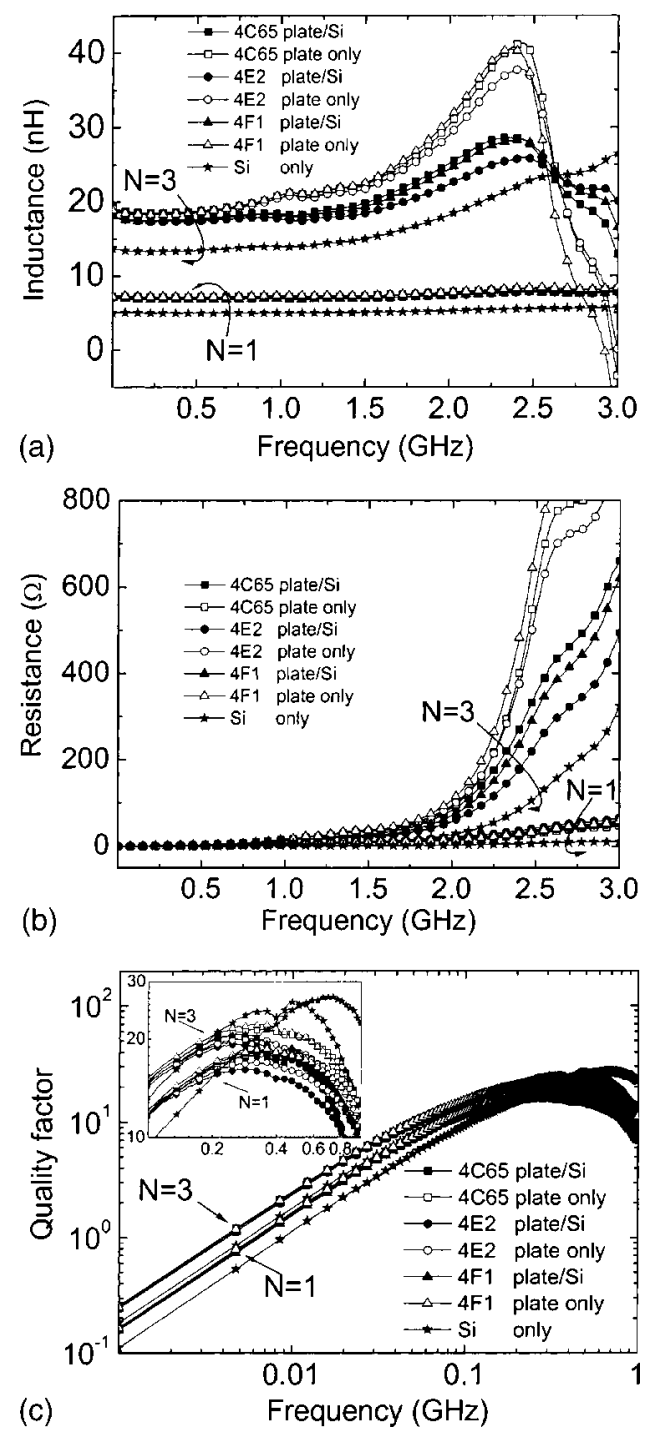

FIG. 3. Frequency dependence of (a) the inductance, (b) the resistance, and (c) the quality factor for the experimental configurations of Fig. 2 using three different ferrites. The coils have an external size $d=0.75 \mathrm{~mm}$ and a number of windings $N=1$ and $N=3$, respectively. The inset of Fig. 3(c) is a zoom on the quality factor behavior at high frequencies.

nantlike behavior: The inductance tends to increase when approaching the resonance frequency $f_{0}=2.5 \mathrm{GHz}$ $\sim 1 / 2 \pi \sqrt{L C_{p}}$, from which we derive an effective parasitic capacitance $C_{p}=0.22 \mathrm{pF}$. Moreover, we observe a somewhat modulated behavior of the inductance at lower frequencies. We think this is due to the distributed capacitance between neighboring coil windings, which tends to short out part of the inductance. Based on the geometrical parameters, we estimate the distributed capacitance per unit length between neighboring windings to be $c_{d}=0.03 \mathrm{pF} / \mathrm{mm}$, consistent with the experimentally determined value for the total $C_{p}$ $\sim \ell c_{d}$, with $\ell=6.34 \mathrm{~mm}$ the total "interwinding" length. The general observation from Fig. 3(a) is the pronounced increase of the inductance (of the order of $40 \%$ ) due to the presence of the ferrite and the strong similarity for the different ferrites used. The reduced inductance values when both the ferrite and the Si substrate are present can be simply explained by eddy current contributions from the $\mathrm{Si}$, which tend to decrease the magnetic flux through the coil.

Figure $3(\mathrm{~b})$ is the frequency-dependent resistance behav- 
ior for the samples of Fig. 3(a), of which the $N=3$ coil most clearly shows the difference between the three configurations. Magnetic losses are present already below $1 \mathrm{GHz}$, but above $2 \mathrm{GHz}$, the ferromagnetic resonance losses of the ferrite plate ("cover only") largely dominate the Si substrate losses. The intermediate resistance values found for the device with both ferrite cover and $\mathrm{Si}$ substrate can be explained by the reduced magnetic losses in the ferrite, due to the eddy currents in the Si that lower the magnetic flux.

Figure 3(c) shows the quality factor $Q=\omega L / R$ of the same set of samples. The strongly increased inductance results in a quality factor enhancement of $25 \%$ with respect to the simple coil up to a frequency of $0.2 \mathrm{GHz}$. At higher frequencies, the ferromagnetic resonance losses of the ferrite decrease the quality factor to a value below that of the device with Si substrate only. The inset of Fig. 3(c) more clearly shows the behavior of the highest- $Q$ values: at around 0.3 $\mathrm{GHz}$, there is a crossing of the family of curves with ferrite cover plate only, and the curves for the coils with both the $\mathrm{Si}$ substrate and ferrite plate present. By this, we demonstrate the limiting frequency for the use of high-resistivity cubic ferrites for rf inductors.

In conclusion, by combining thin plates of commercially available ferrites with postprocessed coils on normal Si substrates, we obtain a $40 \%$ enhancement of the inductance and a $25 \%$ enhancement of the quality factor $(Q=10-20)$ of the realized inductors for frequencies up to $0.2 \mathrm{GHz}$, which represents a limiting frequency for the advantageous use of high-resistivity cubic ferrites in rf inductors. At higher frequencies, ferromagnetic resonance losses in the ferrite domi- nate. Further extension of the useful frequency range would rely on the development of ferrites with increased ferromagnetic resonance frequency.

The authors would like to thank Dr. Victor Fernandez for designing the coils and the staff of the Center of MicroNanoTechnology (CMI) at the Swiss Federal Institute of Technology Lausanne for microfabrication assistance.

${ }^{1}$ G. J. Carchon, W. De Raedt, and E. Beyne, Microelectron. Int. 20, 26 (2003).

${ }^{2}$ K. B. Ashby, I. A. Koullias, W. C. Finley, J. J. Bastek, and S. Moinian, IEEE J. Solid-State Circuits 31, 4 (1996).

${ }^{3}$ H. A. C. Tilmans, K. Baert, A. Verbist, and R. Puers, J. Micromech. Microeng. 6, 122 (1996).

${ }^{4}$ G. W. Dahlmann and E. M. Yeatman, Electron. Lett. 36, 1707 (2000).

${ }^{5}$ M. Yamaguchi, K. Suezawa, K. I. Arai, Y. Takahashi, S. Kikushi, W. D. Li, Y. Shimida, S. Tanabe, and K. Itoh, J. Appl. Phys. 85, 7919 (1999).

${ }^{6}$ M. Yamaguchi, M. Baba, K. Suezawa, T. Moizumi, K. I. Arai, Y. Shimida, A. Haga, S. Tanabe, and K. Itoh, IEEE MTT-S Int. Microwave Symp. Dig. 1, 205 (2000).

${ }^{7}$ M. Yamaguchi, M. Baba, and K.-I. Arai, IEEE Trans. Microwave Theory Tech. 49, 2331 (2001).

${ }^{8}$ S. Chikazumi and S. H. Charap, Physics of Magnetism (Wiley, New York, 1964).

${ }^{9}$ Soft Ferrite and Accessories Catalogue (2002) on http://www.ferroxcube.com

${ }^{10}$ I. Zaquine, H. Benazizi, and J. C. Mage, J. Appl. Phys. 64, 5822 (1988).

${ }^{11}$ J. Y. Park, L. K. Lagorce, and M. G. Allen, IEEE Trans. Magn. 33, 3322 (1997).

${ }^{12}$ M. Abe, Electrochemistry 70, 815 (2002).

${ }^{13}$ Y. Suzuki, R. B. van Dover, E. M. Gyorgy, J. M. Philips, V. Korenivski, D. J. Werder, C. H. Chen, R. J. Cava, J. J. Krajewski, Jr., W. F. Peck, and K. B. Do, Appl. Phys. Lett. 68, 714 (1996).

${ }^{14}$ M. Saidani and M. A. M. Gijs, J. Microelectromech. Syst. 12, 172 (2003). 